\title{
The Statistics of Radio Pulsars: A Spark Model
}

\author{
K.S. Cheng and G.L.Fan \\ The University of Ilong Kong, Pokfulam Road, Hong Kong, China.
}

\begin{abstract}
We investigate pulsar statistics based on theoretical model of radio emission of Ruderman-Sutherland (1975) and the revised spark model of Gil et al. (1999). In the present approach the intrinsic luminosity is no more given by observed relationship between $L_{r}$ and $P, \dot{P}$. Our studies show that R-S \& spark model can explain the statistical properties of radio pulsars reasonably well.
\end{abstract}

\section{Introduction}

It is generally believed that the radio waves are emitted by relativistic outgoing charged particles which are accelerated in polar gap. It is thus natural to suggest that the radio luminosity of pulsars is proportional to the particles current flow and potential drop of gap. We explore the connection between the spark-associated theoretical model - Ruderman \& Sutherland (1975) polar gap model and the revised spark model proposed by J.A. Gil et al. (1999) - and the statistics of radio pulsars.

\section{Brief review of R-S \& spark model}

According to R-S model and the modified R-S model - spark model proposed by Gil et al. (1999), the polar gap is populated by a number of isolated, shortlived discharging sparks. A quasi-central spark driven by $\gamma-\mathbf{B}$ pair production process anchored to the local pole of a complicated surface magnetic field. This polar spark prevents the motion of other sparks towards the pole, restricting them to a circumferencial $\mathbf{E} \times \mathbf{B}$ drift across the planes of field lines converging at the local pole. The polar spark constitutes the core pulsar emission and that the annular rings of drifting sparks contribute to the nested conal components of pulsar beam. The possible observed radio luminosity emitted by sparks depends on the number of rings cutting through by the line of sight.

\section{Monte Carlo simulation of Galactic pulsars and results}

We simulate the distributions of the period, distance, magnetic field and radio luminosity of Galactic pulsars using Monte Carlo method. The simulated statistical properties are compared with the real observed properties of the canonical pulsars by Kolmogorov-Smirnov test. We consider Princeton database (Taylor et al. 1995) and Parkes database (Lyne et al. 1998) respectively. The results of 

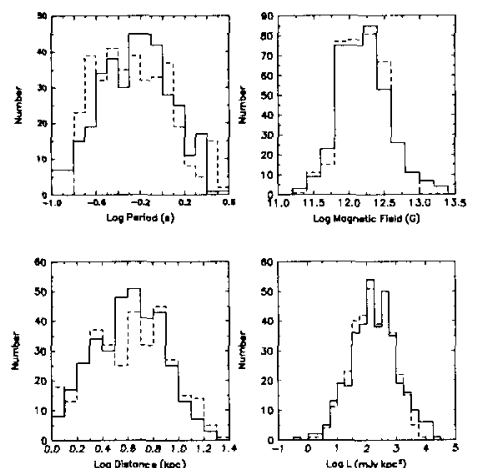

Figure 1. Comparison of the distributions of pulse period, magnetic-field strength, distance and radio luminosity for simulated and Princeton database. The solid lines show the real pulsars, the dash lines the simulated pulsars. The simulated numbers are normalized to the same total as the real pulsars.

K-S test are presented in Table 1 and Fig. 1 illustrates that our simulation can reproduce well the actual observed distribution of the respective properties of the pulsars.

\section{Conclusion and Outlook}

Our study shows that the R-S model and revised spark model proposed by Gil et al. (1999) can be successfully involved in the statistical study of radio pulsars. The observed component distribution can also be conveniently approached by spark model, which we shall discuss in detail in a future publication.

Table 1. K-S test results of the best simulations.

\begin{tabular}{|c|c|c|c|c|}
\hline & Period & Distance & Magnetic Field & Luminosity \\
& $D_{\max }$ Prob & $D_{\max }$ Prob & $D_{\max }$ Prob & $D_{\max }$ Prob \\
\hline Princeton Database & 0.0550 .273 & 0.0650 .125 & 0.0410 .643 & 0.0560 .263 \\
\hline Parkes Database & 0.0870 .102 & 0.0670 .333 & 0.0360 .961 & 0.0730 .241 \\
\hline
\end{tabular}

\section{References}

Gil, J.A., Sendyk, M., \& Cheng, K.S. 1999, MNRAS, in preparation

Lyne, A.G., Manchester, R.N. et al. 1998, MNRAS, 295, 743

Ruderman, M.A., \& Sutherland, P.G. 1975, ApJ, 196,51

Taylor, J.H., Manchester, R.N., Lyne, A.G., \& Camilo, F. 1995, Catalog of 706 Pulsars (Princeton: Princeton Univ. Press.) 